\title{
ReFlections on Five Years of COHERENT TeaChing aCROSS Three Courses on Digital Logic and Computer Systems
}

\author{
Naraig Manjikian \\ Department of Electrical and Computer Engineering, Queen's University, Kingston, Ontario, Canada K7L 3N6 \\ naraig.manjikian@queensu.ca
}

\begin{abstract}
This paper describes a concerted effort over a five-year period to provide a coherent learning experience in a three-course sequence in the areas of digital logic and computer systems. Among other ways, the coherence across the courses stems from having the same instructor, the same laboratory hardware, the same software tools, and the same expectations and approach for assessment of individual learning. Based on the five-year history of academic performance by students on final examinations, the overall outcome is judged as being mixed, where perennial challenges for students in programming have been difficult to address and where other factors such as reduced attendance and interference from other courses have also had adverse effects. To obtain better outcomes, the author recommends higher-level institutional attention to developing more personal learning responsibility in students, as well as more focused efforts to address programming challenges in particular.
\end{abstract}

Keywords: Laboratory activity, course planning and delivery, digital logic, computer systems, programmable logic devices, academic performance by students.

\section{INTRODUCTION}

This paper offers reflections on the experience of the author over five years as the sole instructor for a sequence of laboratory-based courses in digital logic, computer architecture, and interfacing for embedded systems in the Department of Electrical and Computer Engineering at Queen's University. In 2011, the author requested and was granted responsibility as sole instructor for these three courses to revitalize them through adoption of new teaching material and new laboratory equipment. As sole instructor, the author has gone to considerable lengths during the period 2011-2016 to offer a coherent perspective to students on the relevant concepts and their application across these courses. The author has also closely supervised student learning through regular attendance in laboratory sessions and delivery of tutorial sessions in addition to lectures. Finally, author has provided considerable consulting opportunities for students regarding the course material.

The remainder of this paper provides a brief discussion of some related work, an overview of the three courses, a description of the ways in which the sequence is managed coherently, a review of academic performance by students, an assessment of effectiveness, and a summary of conclusions that can be drawn from the effort and its outcomes.

From the perspective of the author, the outcomes reflected in academic performance can be described to be mixed, at best. There are various factors that may counter potential benefits of the coherent approach pursued by the author. As a professional and pedagogical obligation, the delivery of courses should still be pursued in a manner that can, in principle, achieve better results, but ultimately it is the responsibility of each individual student to strive to achieve the benefits. Furthermore, the efforts of a single instructor as discussed in this paper could benefit from higher-level institutional support to address some of the factors that limit the potential benefit.

\section{SAMPLES OF RELATED WORK}

Calazans and Moraes [1] describe their efforts to improve the coherence in teaching two courses related to digital hardware concepts and computer architecture concepts. They focus on using design and implementation of processors in programmable logic as a primary learning approach. From two years of experience, they present student survey results that indicate a reasonably positive reception for their approach. Apart from a brief comment about a slight decrease in the failure rate, no specific academic performance results are provided.

Bezdek et al. [2] describe a more narrowly focused effort for a three-course sequence related to embedded systems, with the key issue being the introduction of the middle course as a new course relative to the first and last in the sequence. This sequence is later in the curriculum, ostensibly after introductory courses on digital logic and computer organization, and includes a graduate-level embedded-systems course as the final element in the se- 
quence. Some information is provided about administrative and other practical challenges faced in the first progression through the sequence, but no academic performance results are provided.

\section{OVERVIEW OF COURSES}

This section provides an overview of the three courses that are the focus of this paper, including some discussion of the history and evolution of the courses. All of the courses are core to both the Electrical Engineering and Computer Engineering programs, and each is a prerequisite for the next in the sequence.

ELEC 271 Digital Systems introduces digital logic circuits and various considerations for design and implementation. The material in the course has been taught for many years (even before the involvement of the author of this paper) using the textbook by Brown and Vranesic [3]. Until 2013, ELEC 271 was a lecture-only course with practical activity pursued in a separate laboratory-only course administered by a different instructor; that laboratory course alternated activity related to ELEC 271 with activity to serve another lecture-only course on basic electric circuits. Beginning in 2014, the laboratory-only course was deleted, and the academic credits for laboratory activity were added to the two relevant courses on electric circuits and digital logic. Commercial hardware with Altera programmable logic devices has been used in all laboratory activity relevant to ELEC 271 for a period extending over two decades.

ELEC 274 Computer Architecture introduces the internal organization of processors, the interconnection of processors with memory and input/output components, and programming in assembly language. ELEC 271 is a prerequisite for ELEC 274 because digital logic concepts are used to describe computer organization. This course has always had a dedicated laboratory component. Since 2012, the textbook by Hamacher et al. [4] has been the sole reference; prior to 2012, earlier editions of the same textbook were used as one of several references by previous instructors. Until 2012, the 8-bit 68HC11 instruction set architecture [5] was the primary vehicle in the laboratory with a custom simulator, but subsequently the 32-bit Nios II architecture [6] was used with commercial hardware using programmable logic to implement computer systems based on this architecture. This change was part of the coherent approach that is the subject of this paper.

ELEC 371 Microprocessor Interfacing and Embedded Systems builds on the foundation of ELEC 271 and ELEC 274 to explore hardware and software considerations in more detail. It has previously been enhanced by Manjikian and Simmons [7] who developed a custom textbook based on the 8-bit $68 \mathrm{HC} 11$ architecture; that textbook was used until 2011. Unlike the simulator-based laboratory activity
ELEC 274 in the period prior to 2012, ELEC 371 had always used $68 \mathrm{HC} 11$-based hardware in the laboratory. In 2012, ELEC 371 transitioned to the 32-bit Nios II architecture in the same manner as ELEC 274. As a consequence, the custom textbook which was based on the $68 \mathrm{HC} 11$ had to be retired, and the textbook by Hamacher et al. [4] was adopted because it includes discussion of hardware/software considerations for embedded systems and system-on-chip implementation in programmable logic devices. Topics in this textbook not covered in ELEC 274 are pursued in ELEC 371.

\section{COHERENT APPROACH}

This section describes how the delivery of the three courses in question has been pursued over five years in a manner to promote coherence with the objective of increasing the efficacy of learning by students. The coherence can be summarized with the following features:

- $\quad$ same instructor for all three courses

- same hardware technology from the same vendor

- $\quad$ same processor architecture for applicable courses

- same commercial and custom software tools

- $\quad$ same textbook for computer-related courses

- $\quad$ same approach for laboratory administration

- $\quad$ same approach for instructor-driven tutorials

- $\quad$ same approach for individual learning assessment Under the coherence reflected in the summary above, the specific objectives have been (a) to exploit consistency for emphasis of fundamental concepts, (b) to strengthen linkages within and across the three courses, (c) to enhance independent learning by students outside the classroom and laboratory, and (d) to address challenges for students in their grasp of concepts related to software and programming due to perennial weaknesses.

In addition to the issues described above, the author pursued efforts in 2011 to develop custom multimedia content for digital systems and computer organization in preparation for the substantial revisions in course material and laboratory hardware for all three courses in the sequence. This initiative has been described in an earlier paper [8]. Development of the multimedia content relates strongly to objective (c) in the preceding paragraph.

The remainder of this section provides some elaboration on certain elements of the coherence that were summarized above. With respect to hardware technology for laboratory activity, all three courses use the commercial Altera DE0 board [9] that is shown in Fig. 1. Altera designed this board to serve educational purposes. The DE0 board was selected by the author in 2011 as part of the transition in the three courses due to its relatively low cost and its simple reliance on a single USB cable connection for power and for communication with an attached desktop computer in the laboratory. By having the same plat- 
form for three successive courses, students should be able to place more attention on learning the fundamentals and applying them more readily using a familiar platform.

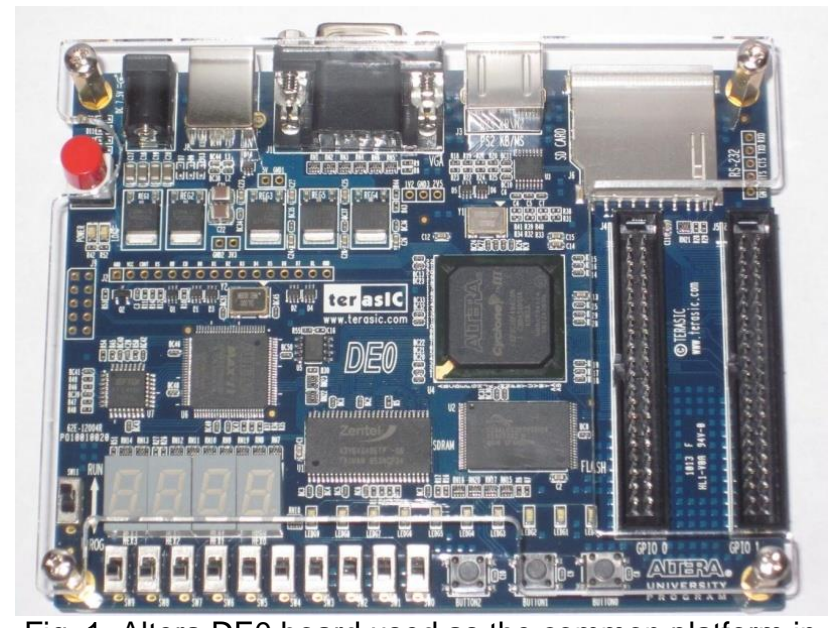

Fig. 1. Altera DE0 board used as the common platform in the laboratory for ELEC 271, ELEC 274, and ELEC 371.

With respect to processor architecture for ELEC 274 and ELEC 371, the Altera Nios II provides a 32-bit platform that is more capable than the 8-bit $68 \mathrm{HC} 11$ platform that had been used until 2011. Furthermore, the Nios II reflects the reduced-instruction-set-computing (RISC) philosophy that leads to a smaller and simpler - yet still complete - set of instructions, relative to the alternative philosophy of having more complex individual instructions. The commonality across courses and relatively simplicity of the instruction set should enable students to devote more attention to learning and applying concepts.

With respect to software tools, having a common commercial platform for all laboratory activity means that students should benefit from having to work with only one complex industrial-calibre tool, Altera Quartus II [10], for logic design and implementation in programmable devices. ELEC 271 is the primary course that utilizes Quartus II, but the author has also had practical activity in ELEC 274 and ELEC 371 that uses lower-level logic to explore processor organization and single-chip computer organization. In addition, Altera provides the Monitor Program [11], which is another software tool designed especially for educational use (i.e., it is not particularly complex) when using the DE0 board in Fig. 1 for computer systems, such as in the laboratory activity for ELEC 274 and ELEC 371. For Quartus II and the Monitor Program, the expectation would be for students to benefit from their use across the sequence of courses.

With respect to a common approach to laboratory administration across the three courses in question, the author as course instructor has provided students with detailed procedures for activity during laboratory sessions due to the nature of the software tools. Furthermore, la- boratory activity has been based on guided preparation with actual code and/or pseudocode provided to students to use for independent learning. Students in each course are typically divided into multiple laboratory sessions, hence the author uses sufficiently distinct session-specific laboratory activity based on the common preparation. The aim is for students to pursue the work in question and learn from that effort, without relying on work completed by classmates in earlier laboratory sessions.

In terms of credit, the author places relatively low weight (approximately 15\%) on the laboratory activity for the overall course mark to emphasize the importance of individual learning by students. From 2011 through to Winter 2016, students were required to work in pairs, but starting in Fall 2016, it was necessary to relax that requirement to allow many groups of three students due to higher enrolment and timetabling with increased demand for shared laboratory space from different courses. The credit earned for each individual laboratory session is split into two equal portions: attendance credit that requires students to arrive within the first 10-15 minutes of 2-3 hour sessions, and credit for successfully completing the session-specific activity. The attendance credit is revoked if a student leaves early with no meaningful completion. Due to the available teaching-assistant resources, the size of classes, and the reality of declining academic integrity for unsupervised work, there are no pre- or postlaboratory written submissions.

With respect to scheduled tutorial activity that complements scheduled lecture activity in the three courses in question, the author as instructor has always delivered tutorials (i.e., not teaching assistants). This conscious choice ensures consistency when reinforcing lecture concepts with posted practice problems. Solutions to such problems are developed and explained during tutorial sessions, typically on the chalkboard and sometimes as code typed and executed live in class. Solutions are intentionally not posted electronically. The aim is to encourage attendance and discussion, or as a minimum to have students take personal responsibility to consult classmates for material missed due to absence from tutorials (and the same expectation certainly applies for lecture absences).

Finally, with respect to learning assessment, it has already been stated above that the author as instructor places relatively low weight on laboratory activity pursued by pairs of students (and now also in groups of three) for calculating the course mark for each student. Consequently, approximately $85 \%$ of the course mark in each of the three courses is determined by supervised assessment of individual learning. Because the three courses in question all involve significant laboratory activity that consumes available teaching-assistant resources, the supervised individual assessment is limited to a single 45-minute inclass quiz at the mid-point of the term (20\% to $25 \%$ of the course mark, depending on the course), and a three-hour 
final examination ( $60 \%$ to $65 \%$ of the course mark). All assessments are closed-book to emphasize individual retention of important details and individual understanding of fundamental concepts and their application. Where appropriate, technical reference information is provided on quiz or examination papers (e.g., a summary of the Nios II instruction set for ELEC 274 and ELEC 371).

Students are provided with considerable learning support in preparation for the supervised individual learning assessment described above in the three courses in question. In addition to practice problems for tutorial sessions, the author as the instructor provides students with prioryear quiz papers along with solutions so that those questions can be attempted for practice. Queen's University instituted a policy twenty years ago to centrally post all prior final examination papers on a university-maintained Website, which provides students with an additional pool of practice questions. With specific awareness of the challenges of the material in ELEC 274 that combines aspects of hardware and software, the author as the instructor has provided weekly 1-2 page practice quizzes (in addition to the practice questions explored during tutorial sessions) with solutions for independent learning. Finally, as documented in an earlier paper [12], custom simulators have been developed by the author for modeling the behavior of simple digital logic circuits and the execution of machine-instruction programs. These simulators are specifically related to the DE0 board and the Nios II instruction set in order for students to pursue independent learning that is directly associated with the material of the courses in question. Students have the ultimate responsibility to use the various examples of support described above in a manner dictated by their learning needs and their available time.

\section{STUDENT ACADEMIC PERFORMANCE AND COMMENTS ON CIRCUMSTANCES}

This section summarizes the academic performance of the students enrolled in the three courses in question from 2011 to 2016 . Furthermore, some context is provided by commenting on various circumstances that, in the opinion of the author as the instructor, may be relevant to understanding the reported performance.

Reflecting the emphasis of the author on individual learning, the academic performance that is reported in this section is based on the average mark for final examinations that cover all of the material in each of the courses in question. The overall course mark also incorporates an earlier individual assessment (mid-term quiz) that only covers the first half of the course, and a contribution from laboratory attendance and completion that may not truly reflect individual grasp of the course material. Comparing the average final examination results with average course marks reveals that the course mark is on average $2 \%$ higher than the final examination mark. Furthermore, the format and difficulty of questions on the examinations (prepared by the same instructor, i.e., the author of this paper) is comparable from year to year. Therefore, the author contends that it is reasonable to use final examination marks as a true reflection of individual learning and for comparisons within the same course across years.

It was stated in the previous section that final examinations from past years are centrally posted by the university for the benefit of students. Given that the author pursued substantial revisions to the three courses considered in this paper, the first group of students taking each revised course was provided with a special practice final examination reflecting the revisions by the author to aid in their preparation and ensure that they were not disadvantaged by being the first group through that revised course. In subsequent years, the official final examination from the first revised offering was available through the university Website, along with the official examinations for all subsequent offerings.

Table 1 summarizes the history of the average marks for final examinations for the three courses in question over several years. The arrows show the prerequisite relationships and the progression of the groups of students through the sequence of the courses (except for a small number of students who must repeat a particular course due to failure and do not obtain a waiver to pursue a successor course with the same group). There are five instances of progression through the three-course sequence in consecutive academic terms between 2011 and 2016, hence the period of interest for the coherent teaching discussed in this paper is considered as being five years.

Below each average mark in Table 1 is a number in parentheses to provide the enrolment in each course as an indication of the growth that the author as sole instructor has confronted since 2011. These three courses are core for students in Electrical and Computer Engineering. In addition, these courses are either core or elective for students in Mathematics and Engineering, and Engineering Physics. Therefore, the number of students typically diminishes in the progression reflected with the arrows.

The summary in Table 1 suggests that the material in ELEC 271 is somewhat more straightforward for students as it is focused on digital logic, but that ELEC 274 presents greater challenges because it combines hardware concepts based on digital logic with programming in assembly language. The perspective of the author as instructor over two decades of teaching is that there are perennial, if not growing, weaknesses related to programming and software development more generally due to such factors as limited exposure prior to entering university. The results in ELEC 371 are generally better than in ELEC 274 due in part to the somewhat higher level of abstraction in 
ELEC 371 for system considerations. Completion of the prerequisite course ELEC 274 may also be beneficial.

Table 1. Averages for final examinations with enrollment in parentheses.

\begin{tabular}{|c|c|c|c|}
\hline Term & $\begin{array}{c}\text { ELEC } \\
271\end{array}$ & $\begin{array}{c}\text { ELEC } \\
274\end{array}$ & $\begin{array}{c}\text { ELEC } \\
371\end{array}$ \\
\hline Fall 2011 & $\begin{array}{c}71 \% \\
(123)\end{array}$ & & \\
\hline Winter 2012 & & $\begin{array}{c}63 \% \\
(103)\end{array}$ & \\
\hline Fall 2012 & $\begin{array}{c}70 \% \\
(132)\end{array}$ & & $\begin{array}{l}69 \% \\
(87)\end{array}$ \\
\hline Winter 2013 & & $\begin{array}{c}55 \% \\
(111)\end{array}$ & \\
\hline Fall 2013 & $\begin{array}{l}81 \% \\
(154) \\
\end{array}$ & & $\begin{array}{l}71 \% \\
(75) \\
\end{array}$ \\
\hline Winter 2014 & & $\begin{array}{l}61 \% \\
(152)\end{array}$ & \\
\hline Fall 2014 & $\begin{array}{l}83 \% \\
(164)\end{array}$ & & $\begin{array}{c}78 \% \\
(123)\end{array}$ \\
\hline Winter 2015 & & $\begin{array}{c}63 \% \\
(166)\end{array}$ & \\
\hline Fall 2015 & $\begin{array}{l}79 \% \\
(207)\end{array}$ & & $\begin{array}{r}79 \% \\
(144)\end{array}$ \\
\hline Winter 2016 & & $\begin{array}{l}59 \% \\
(191)\end{array}$ & \\
\hline Fall 2016 & $\begin{array}{l}77 \% \\
(228)\end{array}$ & & $\begin{array}{r}67 \% \\
(169)\end{array}$ \\
\hline
\end{tabular}

With respect to ELEC 271, the relatively lower marks in Fall 2011 and Fall 2012 can be attributed in part to poor performance on a question worth $15 \%$ on the final examination on the topic of designing finite-state machines, which is covered in the final two weeks of the term. For Fall 2012, in particular, the performance by the students on this topic was particularly poor, with a significant fraction of students leaving blank entire parts of the finite-state-machine question. Beginning in Fall 2013, the author as the instructor made a concerted effort to address waning attention for material at the end of the term with actions such as direct and repeated statements to students about expectations to learn important material throughout the entire course. The better result in Fall 2013 seems to indicate that such actions had a meaningful effect.

With respect to ELEC 274, the academic performance is relatively lower despite a variety of measures to aid students in their learning of the material for this course such as provision of a custom simulator, provision of weekly practice questions with solutions in addition to tutorial problems, and laboratory exercises that cover the essential assembly-language programming concepts and skills that are assessed on the final examination. Not only does the author as the instructor attend essentially all laboratory sessions to offer guidance along with teaching assistants, but the author also provides considerable consulting opportunities for students (e.g., weekly office hours) during the term, and particularly before the midterm quiz and final examination. Unfortunately, relatively few students have effectively exploited such consulting opportunities. The performance results for ELEC 274 in Table 1 suggest that more students should perhaps seek assistance for understanding the course material.

Not only do many students struggle with the hardware/software challenges in ELEC 274, but other potentially challenging courses in the same term such as electronics and electromagnetics may interfere to some extent. It has been necessary, for example, for the author as instructor to intervene in ELEC 274 laboratory sessions and require students to put away their electronics course notes and textbook. Furthermore, there have been patterns of low attendance in ELEC 274 lectures and tutorials, particularly in the second half of the term, as a number of students may elect to devote their time either to other courses or perhaps to non-academic pursuits. For example, during the second half of the Winter 2016 term, the author frequently counted no more than 40-50 students in the classroom out of more than 200 enrolled students. Providing the majority of the ELEC 274 lecture material electronically instead of relying on the chalkboard as in other courses may be a contributing factor. Finally, ELEC 274 is in the Winter term, hence bitter cold, icy sidewalks, and, in some terms, early-morning lectures may deter a number of students from attending class.

With respect to ELEC 371, it has already been stated that students generally perform better than ELEC 274, but certain specific comments can be made about the results for Fall 2016 in Table 1. This group of students in ELEC 371 was the same group of students who displayed particularly poor lecture attendance in ELEC 274 during Winter 2016, and that behavior continued in ELEC 371 (reduced attendance has also been a concern in other years for ELEC 371). During a department meeting in the latter portion of Fall 2016, an instructor of a different third-year course conveyed comments made by students to that instructor on the overwhelming nature of the overall workload during the Fall 2016 term. In any case, this group of students had lower performance on the ELEC 371 final examination than in previous years.

\section{ASSESSMENT OF EFFECTIVENESS}

Based on the results presented in Table 1, and the perspectives of the author as instructor in observing laboratory activity and marking final examinations, the outcomes of the overall effort to enhance coherence across the threecourse sequence can be described as mixed, at best. Some students may recognize that they have undergone an en- 
hanced learning experience, and some students may actually reflect that perception with better performance. For other students, however, there may be little or no benefit. General challenges related to adequate understanding of concepts and especially knowledge retention - within and across courses - are difficult to address and may have worsened over time. These challenges remain particularly acute for aptitude related to software and programming. Despite having provided multimedia content and custom simulators as dynamic and interactive aids for independent learning, the experience of the author has been that the value of such aids has diminished for various reasons, including the manner in which students manage their time. Finally, the experience of the author has been such that student attitudes concerning laboratory activity and its learning benefits have deteriorated over time.

\section{CONCLUSION}

This paper has described a concerted effort to provide coherence across a three-course sequence related to digital logic and computer systems over a period of five years. Based on the academic performance of students over the period in question, the effort can be said to have provided mixed results.

The primary conclusion of the author from this experience is the need for substantially more institutional support (department-level and higher) to strongly instill in students a greater degree of personal learning responsibility and a greater understanding of the objectives of all course sequences, not just the sequence considered in this paper. Without a broader collective vision to address the challenges described in this paper, there will likely be limited benefits from an effort of the type described in this paper by a single instructor for a small subset of courses.

An additional conclusion is that the ongoing challenges of students related to aptitude in programming also need particular attention. Programming concepts and skills are encompassed in at least two other software-oriented courses, including a first-year course for all engineering students. Extending the scope of providing coherence to include those software-oriented courses is an option, albeit a more daunting one.

\section{Acknowledgements}

The initial Altera DE0 boards acquired in 2011 for the coherent revision of the courses described in this paper were purchased with the support of the Better Equipment Donation fund of the undergraduate Engineering Society at Queen's University.

\section{References}

[1] N. L. V. Calazans and F. G. Moraes, "Integrating the teaching of computer organization and architecture with digital hardware design early in undergraduate courses," IEEE Transactions on Education, vol. 44, no. 2, May 2001, pp. 109-119.

[2] M. Bezdek, D. Helvick, R. Mercado, D. Rover, A. Tyagi, and Z. Zhang, "Developing and teaching an integrated series of courses in embedded computer systems," Proceedings of the $36^{\text {th }}$ ASEE/IEEE Frontiers in Education Conference, San Diego, CA, October 2006, pp. T1E19-T1E24.

[3] S. Brown and Z. Vranesic, Fundamentals of Digital Logic with VHDL Design, $3^{\text {rd }}$ edition, McGraw-Hill, 2009.

[4] C. Hamacher, Z. Vranesic, S. Zaky, and N. Manjikian, Computer Organization and Embedded Systems, $6^{\text {th }}$ edition, McGraw-Hill, 2012.

[5] Freescale Semiconductor, M68HC11 Reference Manual, document M68HC11RM/D, revision 6.1, 2007.

[6] Altera Corp., Nios II Classic Processor Reference Guide, document NII5V1, June 2016.

[7] N. Manjikian and S. Simmons, "Evolution and enhancements of a microprocessor systems course," IEEE Transactions on Education, Vol. 42, No. 4, November 1999. Appears in special CD-ROM supplement with article summary on p. 360.

[8] N. Manjikian and V. Sugarman, "Multimedia content and laboratory enhancements for courses in digital systems and computer architecture," Proceedings of the 2013 Canadian Engineering Education Association Conference, Montréal, Québec, June 2013.

[9] Terasic Inc., DEO User Manual, document version 1.6, May 2012.

[10] Altera Corp., Quartus II Handbook Version 13.0 - Volume I: Design and Synthesis, document QII6V1-13.0.0, May 2013.

[11] Altera Corp., Altera Monitor Program Tutorial, document version for Quartus II 13.0, May 2013.

[12] N. Manjikian and V. Sugarman, "Custom graphical simulators for VHDL logic descriptions and the Altera Nios II processor," Proceedings of the 2013 Canadian Engineering Education Association Conference, Montréal, Québec, June 2013. 